\title{
ON THE ISOMORPHISM PROBLEM FOR BURNSIDE RINGS
}

\author{
ROLF BRANDL AND THOMAS HUCKLE
}

(Communicated by Ron Solomon)

Dedicated to Professor H.-J. Vollrath on his sixtieth birthday

\begin{abstract}
Nonisomorphic 3-groups of distinct nilpotency class are constructed with isomorphic Burnside rings.
\end{abstract}

\section{THE RESULT}

W. Burnside in 1911 (see [4, p. $236 \mathrm{ff}$ ) introduced a ring associated to permutation representations of a finite group $G$ which is now called the Burnside ring $\Omega(G)$ of $G$. Indeed, let $M_{1}, \ldots, M_{t}$ be a set of representatives of the transitive permutation representations of $G$. Then every finite $G$-set $M$ decomposes as a disjoint union of transitive $G$-sets, so that we can write $M=\lambda_{1} M_{1}+\cdots+\lambda_{t} M_{t}$ for nonnegative integers $\lambda_{i}$. Moreover, if $M$ and $N$ are two $G$-sets, then there is a natural action of $G$ on the cartesian product $M \times N$ and, by the above, this can be written as an integral linear combination of the $M_{i}$. Allowing negative coefficients (i.e. using the usual Grothendieck construction), this yields a ring-structure on the set of all (generalized) permutation representations of $G$.

The isomorphism problem arises naturally. Indeed, let $G$ and $H$ be finite groups and assume that their Burnside rings $\Omega(G)$ and $\Omega(H)$ are ringisomorphic. What can be said about $G$ if we know $H$ (see the survey article [8] for the analogous problem for group rings). As the additive group of $\Omega(G)$ is free abelian, freely generated by the representatives of transitive $G$-sets, we see that the number $t$ of conjugacy classes of subgroups of $G$ and $H$ are equal. Beyond this trivial remark, a celebrated result of $A$. Dress [5] says that the solubility of $H$ implies solubility of $G$. Furthermore, a number of other properties can be read off. However, see [9].

The ring $\Omega(G)$ is determined by the table of marks $M(G)$ of $G$ (see [7, Chapter 3]). Thus, for the isomorphism problem for Burnside rings, it is of interest to see what properties can be read off from $M(G)$. Note that the table of marks of $G$ determines the poset $\mathscr{C}(G)$ of conjugacy classes of subgroups of $G$ (see [7, p. 120]). The latter contains much less information about $G$. Indeed, all groups of order $p q$ have order-isomorphic posets of conjugacy classes. However, it was shown in [1] that $\mathscr{E}(G) \cong \mathscr{C}(H)$ and $H$ a noncyclic $p$-group

Received by the editors October 15, 1993 and, in revised form, June 16, 1994.

1991 Mathematics Subject Classification. Primary 20B10, 20C10, $20 \mathrm{D} 15$. 
implies that $|G|=|H|$ and if $H$ is abelian or metacyclic, then $G$ and $H$ are isomorphic (see [1] and [3]). Also, the result proved in [2] can be viewed in this context. Indeed, finite groups were classified with precisely one conjugacy class of nonnormal subgroups, and a trivial consequence of this result is that groups of equal order with this property are isomorphic.

It has been conjectured that $\Omega(G) \cong \Omega(H)$ and $H$ a $p$-group implies that $G$ and $H$ are of equal nilpotency class. The objective of this note is to construct a counterexample for this. Indeed, we shall prove:

Theorem. Let $G=\left\langle x, y, z \mid x^{9}=y^{9}=z^{9}=[x, z]=[y, z]=1,[x, y]=z\right\rangle$ and $H=\left\langle x, y, z \mid x^{9}=y^{9}=z^{9}=[y, z]=1,[x, z]=z^{3},[x, y]=z\right\rangle$. Then $\Omega(G) \cong \Omega(H)$. Moreover, $|G|=729=|H|$ and $G$ is nilpotent of class two and $H$ is of class three.

In particular, the nilpotency class of a $p$-group cannot in general be read off from its Burnside ring (at least for $p=3$ ). It seems likely that the analogous construction works for all primes $p \geq 5$. However, it is not clear how to modify the groups for $p=2$. Also, it is not known to us whether $c(G) \leq 2$ implies any bound for the class $c(H)$ of $H$.

\section{THE ISOMORPHISM}

First of all, the groups $G$ and $H$ have been shown in [3] to have isomorphic posets of conjugacy classes, so that they were natural candidates to try. The tables of marks for $G$ and $H$ were calculated using the following sequence of GAP commands:

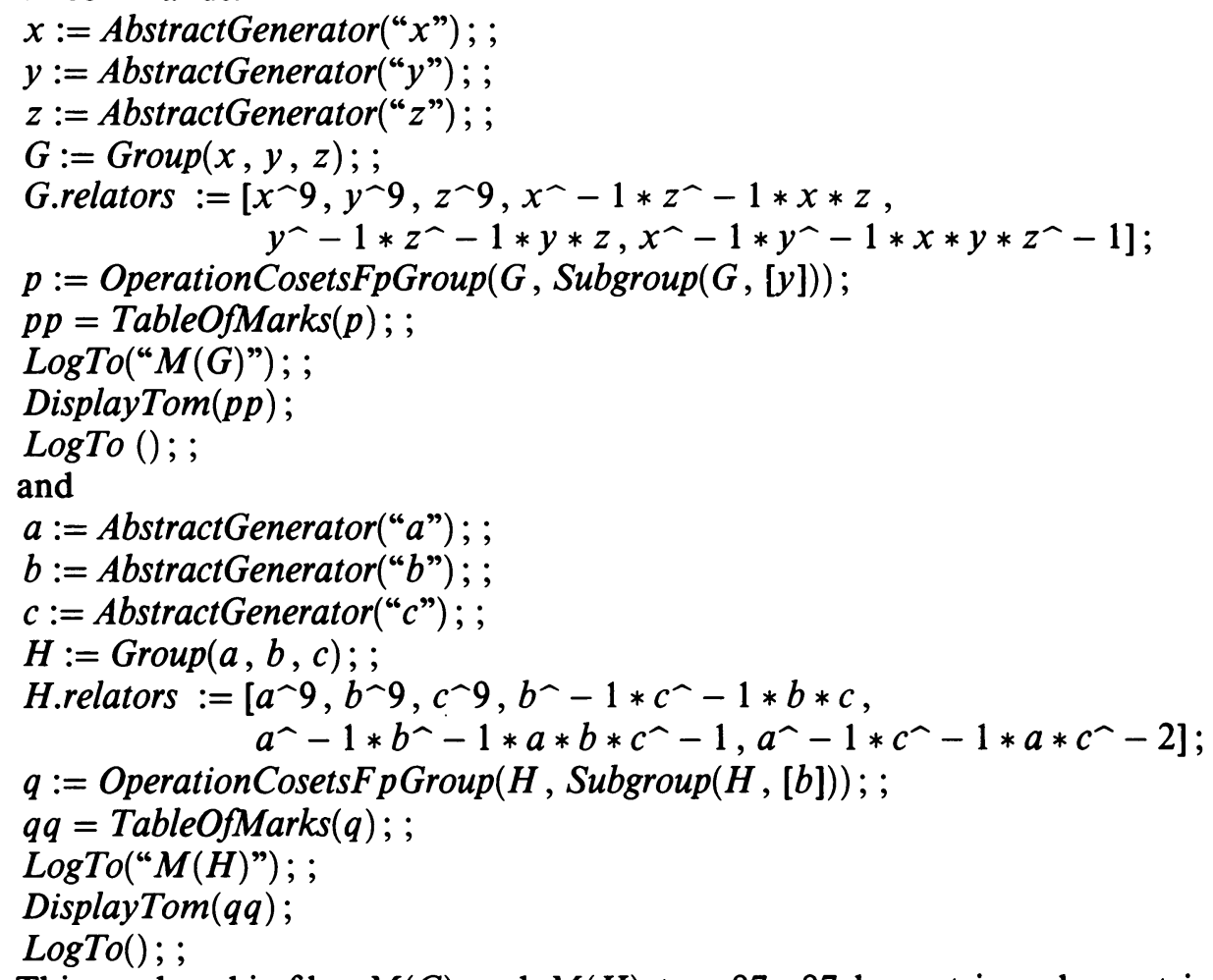

This produced in files $M(G)$ and $M(H)$ two $87 \times 87$ lower triangular matrices that describe the multiplication of the corresponding Burnside rings in terms 
of the representatives of conjugacy classes of subgroups that will be numbered by $1 \ldots 87$. Instead of the LogTo and DisplayTom commands we could also use for example

ppp := MatTom $(p) ;$;

PrintTo(“M(G)", ppp); ;

Clearly, a permutation $\pi$ of the conjugacy classes of subgroups of $H$ does not affect the ring structure of $\Omega(H)$, and our problem was reduced to finding a suitable permutation matrix $P$ related to $\pi$ such that $P^{T} M(H) P=M(G)$.

To boil down the possibilities for $\pi$ for a systematic search, we used the following properties for $G$ and $M(G)$ :

(a) Using CAYLEY at Bologna University, we determined that the automorphism group of $G$ is of order $2^{4} \cdot 3^{9}$ and that $G$ has an automorphism $\varphi$ of order 8 . As $G$ is a two generator 3-group, a result of Burnside [4] on coprime automorphisms says that $\varphi$ acts as an isomorphism of order 8 on the Frattini quotient $G / \Phi(G) \cong Z_{3} \otimes Z_{3}$ of $G$. Hence $\operatorname{Aut}(G)$ acts transitively on the set of maximal subgroups of $G$ and so without loss of generality, the representative 83 corresponding to one of the maximal subgroups of $G$ may be assumed to be fixed.

(b) If the $n \times n$ matrices $M(G)$ and $M(H)$ satisfy

$$
M(G)=P^{T} M(H) P
$$

where $P$ is a permutation matrix, then for all mixed products of the form $q\left(A, A^{T}\right):=A^{k_{1}}\left(A^{m_{1}}\right)^{T} \cdots A^{k_{s}}\left(A^{m_{s}}\right)^{T}$ the analogous relation holds

$$
q\left(M(G), M(G)^{T}\right)=P^{T} q\left(M(H), M(H)^{T}\right) P .
$$

To determine $P$ with (1) we can consider for example $q_{1}\left(A, A^{T}\right):=A^{3}$ or $q_{2}\left(A, A^{T}\right):=A^{2} A^{T}$ and determine properties that must hold for all permutation matrices (2). A solution $P$ of (1) must satisfy such properties, too. Note that the matrices $q_{1}\left(M(G), M(G)^{T}\right)$ have more distinct elements with lower frequency. Thus an inspection of $q_{1}\left(M(G), M(G)^{T}\right)$ provides information about permutations satisfying (1).

(c) For given $n \times n$ matrices $A$ and $B$ we look for a permutation $\pi$ that satisfies $A=P^{T} B P$. Each matrix $A$ and every element $s=A_{i, j}$ of $A$ give rise to a graph $G_{A}(s)$ defined by the knots $P_{1}, \ldots, P_{n}$ and the set of edges $\left\{\left(P_{i}, P_{j}\right) \mid\right.$ if $\left.A_{i, j}=s\right\}$. Permutations applied to $A$ permute the knots of $G_{A}(s)$ in the same way. Hence, $G_{A}(s)$ and $G_{B}(s)$ differ only by the permutation $\pi$. Comparing these two graphs we can extract information about $\pi$. As a trivial example let us consider the case that $s$ appears only once in $A$ and $B$. Then $G_{A}(s)$ is given by one edge $\left\{\left(P_{i}, P_{j}\right)\right\}$ and $G_{B}(s)$ by $\left\{\left(P_{k}, P_{m}\right)\right\}$. Hence, we can deduce that $\pi$ satisfies $\pi(k)=i$ and $\pi(m)=j$.

Combining (a), (b), and (c) one can determine sufficiently many properties of permutation matrices $P$ that satisfy (1). To compute the matrices $q\left(A, A^{T}\right)$ and the related graphs $G_{q\left(A, A^{T}\right)}(s)$ we used MATLAB. We determined one possible permutation $\pi$ that transforms the matrix $M(H)$ into the matrix $M(G)$. This permutation $\pi$ is given by

$$
\begin{aligned}
& (25,26)(30,32)(44,45)(50,51)(56,57)(66,77,72) \\
& (62,71,65,76,70,82,80,75,69,81,64,74,68,79,63,73,67,78) .
\end{aligned}
$$




\section{REFERENCES}

1. R. Brandl, Posets of subgroups in p-groups, Comm. Algebra 20 (1992), 3043-3054.

2. __ Groups with few non-normal subgroups, Comm. Algebra 23 (1995), 2091-2098.

3. R. Brandl and L. Verardi, Metacyclic p-groups and their conjugacy classes of subgroups, Glasgow J. Math. 35 (1993), 339-344.

4. W. Burnside, Theory of groups of finite order, 2nd ed., Cambridge Univ. Press, London and New York, 1911; reprint, Dover, New York, 1955.

5. A. Dress, A characterization of solvable groups, Math. Z. 110 (1969), 213-217.

6. T. O. Hawkes, I. M. Isaacs, and M. Özaydin, On the Möbius function of a finite group, Rocky Mountain J. Math. 19 (1989), 1003-1034.

7. A. Kerber, Algebraic combinatorics via finite group actions, Bibliographisches Institut, Mannheim, 1991.

8. R. Sandling, The isomorphism problem for group rings: A survey, Lecture Notes in Math., vol. 1142, Springer-Verlag, Berlin and New York, 1985, pp. 256-288.

9. J. Thévenaz, Isomorphic Burnside rings, Comm. Algebra 16 (1988), 1945-1947.

(R. Brandl) Mathematisches Institut, Am Hubland 12, D-97074 WÜrzburg, GermanY

E-mail address: brandlevax.rz.uni-wuerzburg.d400.de

(T. Huckle) Institut für Angewandte Mathematik und Statistik, Am Hubland 12, D-97074 WÜRZBURG, GERMANY

E-mail address: huckle@mathematik. uni-wuerzburg.d400.de

(T. Huckle) Department of Computer Science, Stanford University, Stanford, CaliFORNIA 94305 